\title{
Paint Spray Mass Spectrometry for the Detection of Additives from Polymers on Conducting Surfaces
}

\author{
Martin R. L. Paine ${ }^{1}$, Philip J. Barker ${ }^{2}$, and Stephen J. Blanksby ${ }^{1, *}$ \\ ${ }^{I}$ ARC Centre of Excellence for Free Radical Chemistry and Biotechnology School of Chemistry, University of Wollongong, \\ Wollongong NSW 2522, Australia \\ ${ }^{2}$ BlueScope Steel Research, PO Box 202, Port Kembla NSW 2505, Australia
}

Received March 8, 2012; Revised March 12, 2012; Accepted March 13, 2012

First published on the web March 15, 2012; DOI: 10.5478/MSL.2012.3.1.025

\begin{abstract}
Paint Spray is developed as a direct sampling ionisation method for mass spectrometric analysis of additives in polymer-based surface coatings. The technique simply involves applying an external high voltage $(5 \mathrm{kV})$ to the wetted sample placed in front of the mass spectrometer inlet and represents a much simpler ionisation technique compared to those currently available. The capabilities of Paint Spray are demonstrated herein with the detection of four commercially available hindered amine light stabilisers; TINUVIN ${ }^{\circledR} 770$, TINUVIN ${ }^{\circledR} 292$, TINUVIN $^{\circledR} 123$ and TINUVIN $^{\circledR} 152$ directly from thermoset polyester-based coil coatings. Paint Spray requires no sample preparation or pre-treatment and combined with its simplicity - requiring no specialised equipment - makes it ideal for use by non-specialists. The application of Paint Spray for industrial use has significant potential as sample collection from a coil coating production line and Paint Spray ionisation could enable fast quality control screening at high sensitivity.
\end{abstract}

Key words: Paint spray, Additive, Polymer, Ionzation, Mass spectrometry

\section{Introduction}

Recent advances in mass spectrometry (MS) are promoting use of the technology in applications outside traditional laboratory settings. The two major areas of research that are driving these non-traditional MS applications are; (i) the miniaturisation of MS instruments and (ii) the development of ambient ionisation techniques. The deveopment of both portable ${ }^{1}$ and handheld ${ }^{2}$ systems as well as a commercialised field-ready mass spectrometer ${ }^{3}$ represent the progress made in MS miniaturisation over the past 5 years. These advancements have finally enabled mass spectrometric analysis for some highly desirable applications, e.g. on-site detection of toxic chemicals in the environment. ${ }^{4}$ The second major area of research is in the field of ambient ionization or 'direct ionisation' mass spectrometry, which allows information to be obtained instantaneously for analytes at trace-level concentrations. These direct sampling methods, having manifested in the form of almost 30 documented methods, seldom require separation or pre-concentration procedures making them highly amenable to rapid analysis and high-throughput screening applications. ${ }^{5-8}$ Each method is aimed at the direct sampling of analytes in untreated samples allowing examination in their ambient state and is critical to allow untrained

\footnotetext{
*Reprint requests to Assoc. Prof. Stephen J. Blanksby

E-mail: blanksby@uow.edu.au
}

practitioners, such as clinical personnel or first responders, to use MS equipment in situ and obtain immediate results.

Ambient ionisation mass spectrometry has been shown to be effective for the direct analysis of a wide range of compounds within chemical, ${ }^{9,10}$ biological ${ }^{11-13}$ and forensic applications. $^{14,15}$ Recently, the ambient MS methods of desorption electrospray ionisation (DESI) and liquid extraction surface analysis (LESA) have been employed for the analysis of polymers, including the detection and characterisation of hindered amine light stabilisers (HALS) in thermoset polyester-based coil coatings. ${ }^{16,17}$ HALS represent a class of polymer additive that act as free radical scavenging antioxidants present in the formulation of many plastics and surface coatings. ${ }^{18}$ Initial oxidation of the hindered amine yields a stable nitroxyl radical that is proposed to react with macro alkyl radicals generated in situ from polymer degradation. Further reaction of this polymer-bound hindered amine with a polymer peroxyl radical leads to termination of these self-propagating radical species to even-electron products and regenerates the active nitroxyl radical. A key feature of the purported cyclic mechanism is the regenerative, non-sacrificial nature of the nitroxyl radical. This is consistent with the high efficacy observed with HALS in most applications. However, the protection afforded by HALS decreases over time suggesting there are competing processes involved that lead to either deactivation or volatilisation of these additives.

While DESI and LESA have both demonstrated their use as potent analytical tools they both require specialised ion 
source configurations. In collaboration with our industry partners our aim is to develop a simpler, more robust method suitable for process monitoring that does not require such specialised equipment. Ideally, this method should be able to be carried out on any mass spectrometer with an atmospheric pressure ion source interface. The present study reports a method that builds on the paper spray ionisation method developed by the research groups of Cooks, Ouyang and co-workers at Purdue University (West Lafayette, Indiana, USA). ${ }^{19,20}$ The direct sampling method, reported herein as 'Paint Spray', shares characteristics of both ESI and the ambient ionisation methods and is useful for fast, qualitative analysis of polymer-based surface coatings on conductive surfaces. Analyte desorption is achieved by liquid extraction of analytes at or near the surface, and a high electric field is used to facilitate ionisation. Pneumatic assistance is not required to transfer the analyte: a voltage is simply applied to the wetted metal substrate, which is positioned in front of a mass spectrometer.

\section{Experimental}

\section{Reagents}

Methanol and formic acid were high performance liquid chromatography grade (Crown Scientific, Minto NSW, Australia). The hindered amine light stabilisers TINUVIN ${ }^{\circledR} 770$ (bis (2,2,6,6-tetramethyl-4-piperidinyl) sebacate), TINUVIN ${ }^{\circledR}$ 292 (bis (1,2,2,6,6-pentamethyl-4-piperidinyl) sebacate), TINUVIN $^{\circledR} 123$ (bis (1-octyloxy-2,2,6,6-tetramethyl-4piperidinyl) sebacate) and TINUVIN ${ }^{\circledR} 152(2,4$-bis $(N$-butyl- $N$ (1-cyclohexyloxy-2,2,6,6-tetramethyl-4-piperidinyl)amino)6-(2-hydroxyethylamine)-1,3,5-triazine) supplied by Ciba Specialty Chemicals (Basel, Switzerland) and were used without purification (Figure 1).

\section{Preparation of coated steel panels}

The topcoat paint system employed in these studies was a solvent-borne, polyester topcoat paint incorporating a melamine-formaldehyde cross-linker, acid catalysed and

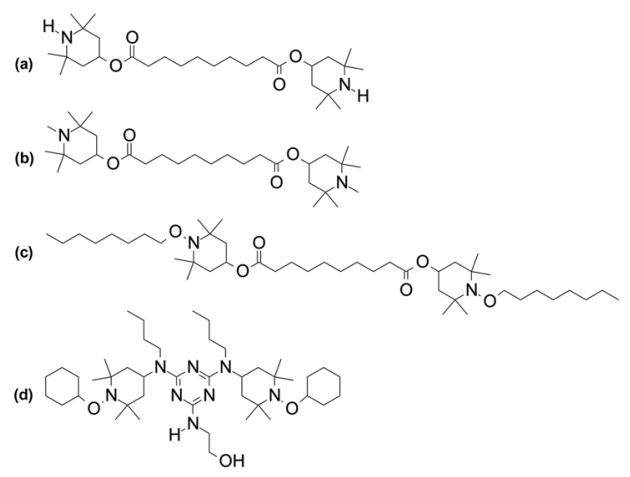

Figure 1. Structures of the four commercially available hindered amine light stabilisers used in this study: (a) TINUVIN ${ }^{\circledR} 770$, (b) TINUVIN $^{\circledR} 292$, (c) TINUVIN ${ }^{\circledR} 123$ and (d) TINUVIN ${ }^{\circledR} 152$. formulated for coil paint-line application. As a wet paint, this sample was found to be $40 \% \mathrm{w} / \mathrm{w}$ resin solids by thermogravimetry (Perkin-Elmer TGA 7, Perkin-Elmer Co. Ltd., Norwalk CT, USA). A bulk sample of this paint was sub-sampled and weighed into small containers with HALS added at $c a$. $2 \%$ (based upon total resin solids). The coated samples were laboratory prepared upon pre-primed (commercial chromated polyester primer) panels of a $0.6 \mathrm{~mm}$ thick GALVALUME $^{\mathbb{R}}$-type steel substrate. Wet paint was applied using a \#28 wire-wound draw-down bar then cured for 55 seconds in a fan forced oven set at $262^{\circ} \mathrm{C}$. Under these conditions, 55 seconds equates to a peak metal temperature of $232^{\circ} \mathrm{C}$. The panels were then cut into small sections $(25 \times 75 \mathrm{~mm})$ using hydraulic shears.

\section{Paint spray}

All experiments were carried out with a ThermoFinnigan LTQ 2-dimensional (2-D) linear ion trap mass spectrometer (ThermoFisher, San Jose CA, USA). Instrumental conditions used for positive-ion mode mass spectrometry: Paint Spray voltage: $5 \mathrm{kV}$; capillary temperature: $200^{\circ} \mathrm{C}$; heated-capillary voltage: $30 \mathrm{~V}$; tube-lens voltage: $100 \mathrm{~V}$. Paint Spray-MS/MS spectra were acquired under the same experimental conditions with a precursor ion isolation width of $1.5 \mathrm{Da}$ and normalised collision-induced dissociation energy of 30 (arb. units). Metal panels with a thermosetting polyesterbased coating were fixed in position in front of the mass spectrometer inlet with a corner directed toward the extraction orifice. Small volumes of methanol $(10 \mu \mathrm{L})$ acidified with formic acid $(0.1 \% \mathrm{v} / \mathrm{v})$ were deposited on the coating at the point closest to the mass spectrometer and a high voltage $(5 \mathrm{kV})$ was applied to the coating using a crocodile-clip connection (Figure 2).

\section{Results and Discussion}

Figure 2 shows the experimental set-up for Paint Spray-MS with samples connected to a high voltage using a crocodile-clip and mounted in front of the MS inlet. Preliminary Paint Spray-MS analysis of coatings before wetting the surface with acidified methanol yielded no detectable signal for HALS from the sample. Following addition of solvent,

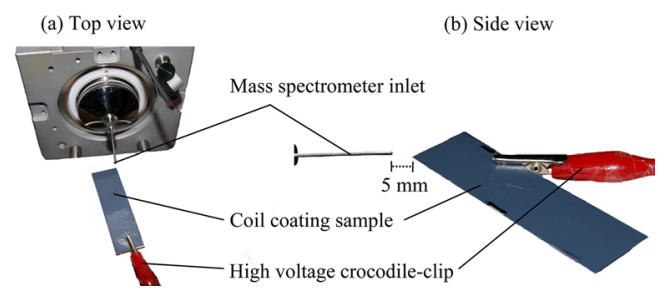

Figure 2. Photographic representations of the experimental setup for Paint Spray ionisation as seen from; (a) the top view and (b) the side view showing the alignment of the mass spectrometer inlet with the coil coating sample and the position of the high voltage crocodile-clip. 
however, Paint Spray-MS provided rapid detection of HALS from the sample with high signal intensities. Samples were positioned on-axis with the MS inlet at a distance of $5 \mathrm{~mm}$ with no signal detected by the mass spectrometer at distances $>10 \mathrm{~mm}$. The corner of the sample was directed at the MS inlet as the high electric potential applied between the sample $(5 \mathrm{kV})$ and the MS inlet $(30 \mathrm{~V})$ generates an electric field that induces a charge that accumulates at the apex of the sample. An insulating substrate (i.e., a glass microscope slide) was also tested but was unable to generate ions using this configuration.

The addition of solvent to the samples surface coincided with the production of a visible droplet stream similar to that produced by the Taylor cone observed under electrospray ionisation conditions. The potential difference and pressure gradient serve to draw the charged droplets into the mass spectrometer with the transition from atmospheric pressure to high vacuum inside the mass spectrometer enough to facilitate analyte desolvation. A constant droplet stream was generated for up to 30 seconds with $10 \mu \mathrm{L}$ of solvent deposited with droplet generation and analyte detection reproducible on the addition of subsequent $10 \mu \mathrm{L}$ aliquots of solvent.

Positive ion Paint Spray-MS spectra of four polyesterbased coil coatings each containing a different commercially available hindered amine light stabilisers are shown in Figure 3. The spectra yield intense signals for singly and/or doubly charged ions arising from protonation of the heterocyclic nitrogen(s) within the HALS compounds. In comparison with other ambient mass spectrometric methods employing solvent based desorption, Paint Spray-MS spectra closely resemble those acquired using LESA and differ to that of DESI in terms singly/doubly charged ion abundance ratios. This can be rationalised by the relatively large amount of solvent involved with LESA and Paint-Spray-MS $(2-10 \mu \mathrm{L})$ compared with DESI (microdroplets). Figure 3(a) shows the positive ion Paint Spray-MS spectrum for a thermoset coil coating formulated with TINUVIN ${ }^{\circledR}$ 770. The spectrum exhibits a base peak at $\mathrm{m} / z 241.4$ that corresponds to the $[\mathrm{M}+2 \mathrm{H}]^{2+}$ ion of TINUVIN ${ }^{\circledR} 770$ with no other ion detected at a relative abundance higher than $10 \%$. A small peak at $m / z 481.4$ (5\% relative abundance) is also observed that corresponds to the $[\mathrm{M}+\mathrm{H}]^{+}$ion of TINUVIN ${ }^{\mathbb{R}} 770$. Figure $3(\mathrm{~b})$ shows the positive ion Paint Spray-MS spectrum for a thermoset coil coating formulated with TINUVIN ${ }^{\circledR} 292$. The spectrum exhibits a base peak at $\mathrm{m} / z$ 255.5 that corresponds to the $[\mathrm{M}+2 \mathrm{H}]^{2+}$ ion of TINUVIN $^{\mathbb{R}} 292$ and is clearly distinguishable within the spectrum with no other ion detected at a relative abundance higher than $25 \%$. A small peak at $\mathrm{m} / \mathrm{z} 509.5$ (5\% relative abundance) is also observed that corresponds to the $[\mathrm{M}+\mathrm{H}]^{+}$ion of TINUVIN ${ }^{\circledR}$ 292. Figures 3(a) and (b) are both dominated by the doubly charged ion of the precursor HALS compound present. This can be rationalised by the high basicity of the heterocyclic nitrogens present for these compounds with TINUVIN ${ }^{\circledR} 770$ $\left(\mathrm{pK}_{\mathrm{b}}\right.$ 5.0) containing two secondary amines and TINUVIN ${ }^{\circledR}$ $292\left(\mathrm{pK}_{\mathrm{b}} \sim 5.0\right)$ containing two tertiary amines. ${ }^{21}$ These two compounds therefore have a greater propensity to protonate at both functional nitrogen groups.

Figure 3(c) shows the positive ion Paint Spray-MS spectrum for a thermoset coil coating formulated with TINUVIN $^{\circledR} 123$. The spectrum exhibits two major peaks at $m / z 369.5$ and 737.5 that correspond to the $[\mathrm{M}+2 \mathrm{H}]^{2+}$ and $[\mathrm{M}+\mathrm{H}]^{+}$ions for TINUVIN ${ }^{\circledR} 123$, respectively. Unlike the previous two examples, TINUVIN ${ }^{\circledR} 123$ has been optimised for use in acid-catalysed polymer systems with the heterocyclic nitrogens present modified to alkoxyamines
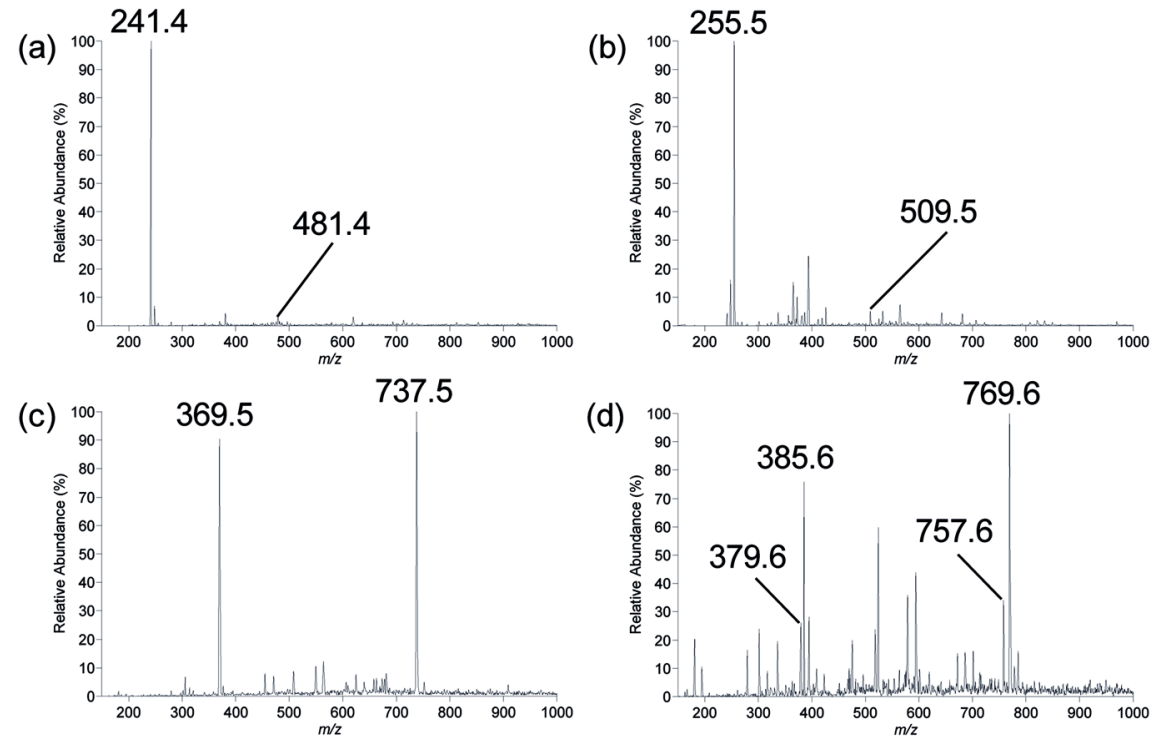

Figure 3. Positive ion Paint Spray-MS spectra of four polyester-based thermoset coil coating samples formulated with either; (a) TINUVIN $^{\circledR} 770$, (b) TINUVIN ${ }^{\circledR} 292$, (c) TINUVIN $^{\circledR} 123$, or (d) TINUVIN $^{\circledR} 152$. 
(cf. Figure 1(c)). This structural modification decreases the basicity of TINUVIN ${ }^{\circledR} 123$, reducing interference with the curing process and limits interactions with other polymer components. The change in acidity is highlighted by the increase in the ratio of singly-to-doubly charged ions detected in Figure 3(c).

The three stabilisers reported thus far can all be considered as 'migratory' HALS, able to move freely through the polymer matrix when the polymer is above its glass transition temperature. Above this temperature, the polymers physical properties become altered, changing from a hard and relatively brittle state into a molten or rubber-like state. Figure 3(d) is a positive ion Paint Spray-MS spectrum of the 'non-migratory' HALS TINUVIN ${ }^{\circledR}$ 152. This compound is designed to co-condense with the polymer backbone through condensation of the primary alcohol substituent to melamine and isocyanate cross-linkers and therefore should not be able to be detected under normal ambient ionisation conditions. However, Figure 3(d) exhibits ions at $\mathrm{m} / \mathrm{z} 379.6$ and 757.6 that correspond to the $[\mathrm{M}+2 \mathrm{H}]^{2+}$ and $[\mathrm{M}+\mathrm{H}]^{+}$ ions of TINUVIN ${ }^{\circledR} 152$ indicating some of the HALS has remained unbound after the curing process. Also, two major peaks were detected at $\mathrm{m} / \mathrm{z} 385.6$ and 769.6 that correspond to what is proposed to be a synthetic byproduct. Tentative structural elucidation of the ion at $\mathrm{m} / \mathrm{z}$ 769.5 using Paint Spray-MS/MS experiments (data not shown) indicates that the aminoethanol group functionalised to the triazine has been modified to a butylamino, rendering it unable to co-condense with the polymer.

\section{Conclusions}

Paint Spray ionisation has been developed for direct MS analysis of polymer-based surface coatings requiring no sample pre-treatment. Its capabilities have been demonstrated with the analysis of four commercially available HALS (three migratory and one non-migratory) present in polyester-based thermoset coil coatings. For the three migratory HALS, the singly or doubly charged ion of the parent HALS compound were detected as the base peak, being clearly distinguishable within the spectrum. For the non-migratory HALS $\left(\right.$ TINUVIN $^{\circledR} 152$ ), the $[\mathrm{M}+\mathrm{H}]^{+}$ ion of a synthetic by-product unable to co-condense with the polymer was detected as the base peak. Unreacted TINUVIN $^{\circledR} 152$ present in the coating was also detected as both a singly and doubly charged ion. The technique has proven to be rapid and simple to employ making it ideal for use by those outside the research laboratory setting. The potential of Paint Spray ionisation when combined with a portable or handheld mass spectrometer for mass analysis expands the possibilities for MS, such as on-line quality control within the surface coating industry. For instance, the degree of cross-linking of non-migratory HALS could be monitored by the detection of the unbound material present in the coating.

\section{Acknowledgements}

M.R.L.P. and S.J.B. acknowledge funding from the Australian Research Council (ARC) and BlueScope Steel (LP0775032) and support from the ARC Centre of Excellence For Free Radical Chemistry and Biotechnology (CE0561607). M.R.L.P. is the holder of an Australian Postgraduate Award (Industry).

\section{References}

1. Mulligan, C. C.; Talaty, N.; Cooks, R. G. Chem. Commun. 2006, 1709.

2. Keil, A.; Talaty, N.; Janfelt, C.; Noll, R. J.; Gao, L.; Ouyang, Z.; Cooks, R. G. Anal. Chem. 2007, 79, 7734.

3. Wells, J. M.; Roth, M. J.; Keil, A. D.; Grossenbacher, J. W.; Justes, D. R.; Patterson, G. E.; Barket Jr, D. J. J. Am. Soc. Mass Spectrom. 2008, 19, 1419.

4. Huang, G.; Gao, L.; Duncan, J.; Harper, J.; Sanders, N.; Ouyang, Z.; Cooks, R. J. Am. Soc. Mass Spectrom. 2009, $21,132$.

5. Harris, G. A.; Galhena, A. S.; Fernandez, F. M. Anal. Chem. 2011, 83, 4508.

6. Cooks, R. G.; Ouyang, Z.; Takats, Z.; Wiseman, J. M. Science 2006, 311, 1566.

7. Harris, G. A.; Nyadong, L.; Fernandez, F. M. Analyst 2008, 133, 1297.

8. Weston, D. J. Analyst 2010, 135, 661.

9. Weston, D. J.; Bateman, R.; Wilson, I. D.; Wood, T. R.; Creaser, C. S. Anal. Chem. 2005, 77, 7572.

10. Hopfgartner, G.; Bourgogne, E. Mass Spectrom. Rev. 2003, 22, 195.

11. Shiea, J.; Huang, M. Z.; Hsu, H. J.; Lee, C. Y.; Yuan, C. H.; Beech, I.; Sunner, J. Rapid Commun. Mass Spectrom. 2005, 19, 3701.

12. Nemes, P.; Vertes, A. Anal. Chem. 2007, 79, 8098.

13. Thunig, J.; Flø, L.; Pedersen-Bjergaard, S.; Hansen, S. H.; Janfelt, C. Rapid Commun. Mass Spectrom. 2012, 26, 133.

14. Ifa, D. R.; Jackson, A. U.; Paglia, G.; Cooks, R. G. Anal. Bioanal. Chem. 2009, 394, 1995.

15. Takats, Z.; Cotte-Rodriguez, I.; Talaty, N.; Chen, H.; Cooks, R. G. Chem. Commun. 2005, 1950.

16. Paine, M. R. L.; Barker, P. J.; Blanksby, S. J. Analyst 2011, 136, 904.

17. Paine, M. R. L.; Barker, P. J.; Maclauglin, S. A.; Mitchell, T. W.; Blanksby, S. J. Rapid Commun. Mass Spectrom. 2012, 26, 412 .

18. Hodgson, J. L.; Coote, M. L. Macromolecules 2010, $43,4573$.

19. Liu, J.; Wang, H.; Manicke, N. E.; Lin, J.-M.; Cooks, R. G.; Ouyang, Z. Anal. Chem. 2010, 82, 2463.

20. Wang, H.; Liu, J.; Cooks, R. G.; Ouyang, Z. Angew Chem. Int. Ed. 2010, 49, 877.

21. Schaller, C.; Rogez, D.; Braig, A. J. Coat. Technol. Res. 2009, 6,81 . 